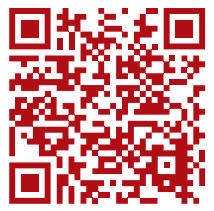

\title{
Comparación de la efectividad en la analgesia postoperatoria con el uso de anestésico local en la solución tumescente, ropivacaína vs lidocaína
}

\author{
Comparison of the effectiveness in postoperative analgesia with the use of \\ local anesthetic in the tumescent solution, ropivacaine vs lidocaine \\ Dra. Sonia Leslie Fuentes-Trejo,* Dra. Elizabeth María Brugés-Sánchez, ${ }^{\ddagger}$ \\ Dr. José Fernando Lara-Calzada, ${ }^{\S}$ Dr. Jorge Eduardo Krasovsky-Santamarina, ${ }^{\natural}$ \\ Dr. Javier Rivas-Jiménez, ${ }^{\ddagger, \|}$ Dra. Carla Mónica Encinas-Pórcel ${ }^{\S, * *}$
}

Palabras clave:

Anestésico local, ropivacaína,

lidocaína, liposucción, dolor, analgesia postoperatoria.

Keywords: Local anesthetic, ropivacaine, lidocaine, liposuction, pain, postoperative analgesia.

\footnotetext{
* Médico Especialista en Anestesiología. Facultad Mexicana de Medicina de la Universidad La Salle. Hospital Ángeles Pedregal.

* Médico Especialista en Cirugía Plástica, Estética y Reconstructiva.

$\S$ Médico Especialista en Anestesiología del Hospital Ángeles Pedregal.

" Médico Especialista en Cirugía Plástica, Estética y Reconstructiva del Hospital Ángeles Pedregal. Director Médico de la Clínica Berlín Médica Boutique.

॥ Centro Médico

Nacional 20 de

Noviembre, ISSSTE.

** Jefe de Servicio de Quirófano del Hospital San Ángel Inn Sur.
}

Clínica Berlín Médica Boutique. México.

\section{RESUMEN}

En el acto quirúrgico los anestésicos locales forman parte de la analgesia preventiva para evitar la sensibilidad central y periférica. El adecuado control del dolor postoperatorio reduce la morbimortalidad, brinda bienestar físico y emocional e inhibe reflejos nociceptivos, lo que permite una adecuada dinámica ventilatoria y movilización temprana. El dolor postoperatorio en la liposucción es subestimado y hasta $27.4 \%$ de los pacientes requieren tramadol. Los objetivos de este trabajo son demostrar que la adición de ropivacaína a las soluciones de infiltración confiere mayor analgesia postoperatoria que la lidocaína; comparar la duración del efecto analgésico de ambos anestésicos locales y determinar si el uso de la ropivacaína reduce la medicación analgésica de rescate. Se incluyeron 53 pacientes en una cohorte prospectiva, $49 \%$ fueron tratados con lidocaína y $51 \%$ con ropivacaína. Los pacientes del grupo de ropivacaína presentaron una menor incidencia de dolor postoperatorio $(\mathrm{p}=0.039)$. La aparición de dolor para los pacientes con lidocaína fue a partir de las primeras cuatro horas y para la ropivacaína se acota a partir de las 12 horas ( $p=0.024)$. Se estimó que la cantidad de horas que transcurrieron hasta solicitar la medicación de rescate fue de 9.87 horas para el caso de la lidocaína y de 11.13 horas para la ropivacaína. La adición de ropivacaína a las soluciones de infiltración para la cirugía de contorno corporal es una estrategia prometedora para el manejo del dolor postoperatorio. Sin embargo, se requieren más estudios para corroborarlo.

\section{ABSTRACT}

During surgical procedures, local anesthetics are part of the preventive analgesia to circumvent central and peripheral sensitivity. Adequate control of postoperative pain reduces morbidity and mortality, provides physical and emotional comfort, and inhibits nociceptive reflexes, which allows adequate ventilatory dynamics and early mobilization. Postoperative pain in liposuction is underestimated and up to $27.4 \%$ of patients require tramadol. The objectives of this work are to demonstrate that the addition of ropivacaine to infiltration solutions confers greater postoperative analgesia than lidocaine; to compare the duration of the analgesic effect of both local anesthetics and to determine if the use of ropivacaine reduces rescue analgesic medication. 53 patients were included in a prospective cohort; $49 \%$ were treated with lidocaine and $51 \%$ with ropivacaine. The patients in the ropivacaine group had a lower incidence of postoperative pain ( $p=0.039)$. The appearance of pain for patients treated with lidocaine was started the first 4 hours on and for ropivacaine it was limited from 12 hours ( $p=$ 0.024). The number of hours that elapsed before requesting rescue medication was estimated to be 9.87 hours for lidocaine and 11.13 hours for ropivacaine. The addition of ropivacaine to infiltration solutions for body contouring surgery is a promising strategy for postoperative pain management. More studies are required to corroborate this.

Citar como: Fuentes-Trejo SL, Brugés-Sánchez EM, Lara-Calzada JF, Krasovsky-Santamarina JE, Rivas-Jiménez J, Encinas-Pórcel CM. Comparación de la efectividad en la analgesia postoperatoria con el uso de anestésico local en la solución tumescente, ropivacaína vs lidocaína. Cir Plast. 2021; 31 (3): 102-106. https://dx.doi.org/10.35366/103711 
Recibido:

20 septiembre 2021

Aceptado:

18 octubre 2021

\section{INTRODUCCIÓN}

L as cirugías plásticas tienen un incremento anual entre 5 y 10\%. En 2016, Estados Unidos, Brasil, Japón, Italia y México conformaron el $41.4 \%$ del total de procedimientos quirúrgicos y cosméticos. La liposucción es el segundo procedimiento más popular, sólo en el año 2016 se reportaron 1'453,340 procedimientos, de los cuales 304,850 se realizaron en Estados Unidos y 69,445 en México. ${ }^{1}$

A partir de la introducción de la solución de Klein, ${ }^{2}$ se incorporaron diferentes combinaciones de fármacos en las soluciones de infiltración; ${ }^{3}$ posteriormente se desarrollaron diferentes técnicas, entre las que destaca la súper húmeda, por ser la que mejor relación tiene en cuanto a volumen de infiltración y drenaje. ${ }^{4}$ Los preparados de las soluciones incluyen tradicionalmente lidocaína y epinefrina a distintas concentraciones. ${ }^{5,6}$

La lidocaína, al igual que el resto de los anestésicos locales, actúa a través del bloqueo de canales de sodio de neuronas periféricas sensitivas, lo que impide la conducción nerviosa. Se considera una base débil; se une $70 \%$ a proteínas plasmáticas y a altas concentraciones plasmáticas $(10 \mu \mathrm{g} / \mathrm{mL})$, también se une a la albúmina y tiende a causar efectos adversos. Los síntomas de toxicidad en orden de aparición son: mareo, fasciculaciones, tinnitus, parestesias, convulsiones, coma y paro cardiorrespiratorio. Prácticamente todo el fármaco es metabolizado en el hígado antes de ser excretado. ${ }^{7}$ La ropivacaína es un enantiómero S puro, altamente soluble a los lípidos; su propiedad más característica es la prolongada duración de acción, tiene metabolismo hepático y $86 \%$ de eliminación renal. Los síntomas de toxicidad son similares a los de la lidocaína y en el mismo orden de aparición. Los enantiómeros $\mathrm{S}$ no protegen de los accidentes cardiacos, pero permiten una reanimación más eficaz. ${ }^{8}$

La toxicidad sistémica de los anestésicos locales está directamente relacionada con la concentración sérica del fármaco mientras se absorbe en la circulación; está determinada por la dosis, el sitio y la forma de administración. La dosis máxima de los anestésicos locales, ya sea tópica o inyectada, se incrementa cuando se agrega un agente vasoconstrictor (adrenalina).
La concentración sérica aumenta a un ritmo más lento cuando el flujo sanguíneo disminuye en el sitio de aplicación mientras el anestésico es retenido. Este proceso incrementa la duración del efecto analgésico.

La dosis máxima para infiltración subcutánea de lidocaína con vasoconstrictor es de 6-7 $\mathrm{mg} / \mathrm{kg}$ de peso (sin exceder $500 \mathrm{mg}$ por dosis) y la duración del efecto es de 120-240 minutos (con epinefrina); para el caso de la ropivacaína con vasoconstrictor, es de 3-4 mg/kg de peso (sin exceder $225 \mathrm{mg}$ por dosis) y la duración del efecto es de 180-480 minutos (con epinefrina). ${ }^{9}$

Los anestésicos locales son parte de la analgesia preventiva, cuyo objetivo es evitar la sensibilidad central y periférica, así como la posible evolución del dolor crónico después de la manipulación de tejidos en el acto quirúrgico y la disminución del consumo de medicamentos. El manejo inadecuado del dolor puede desencadenar alteraciones cardiovasculares, respiratorias, gastrointestinales, urinarias, neuroendocrinas y psicológicas. Un adecuado control del dolor reduce significativamente la morbimortalidad, brinda bienestar físico y emocional e inhibe reflejos nociceptivos, lo que permite una adecuada dinámica ventilatoria y movilización temprana. ${ }^{10,11}$

El dolor postoperatorio en la liposucción es generalmente subestimado, hasta $27.4 \%$ de los pacientes requieren tramadol y $4.3 \%$ opioides más potentes. ${ }^{12}$ Ante la actual crisis en el abuso de opioides y muertes por sobredosis, gran parte alimentadas por analgésicos opioides recetados, es necesario el desarrollo de nuevas estrategias para el control del dolor que igualen o excedan la efectividad de estos medicamentos con menos efectos secundarios adictivos y letales, como la depresión respiratoria. ${ }^{13}$

El objetivo de este trabajo fue demostrar que la adición de ropivacaína a las soluciones de infiltración en liposucción confiere mayor analgesia postoperatoria que la lidocaína, comparar la duración del efecto analgésico de ambos anestésicos locales y determinar si el uso de ropivacaína reduce la medicación analgésica de rescate.

\section{MATERIAL Y MÉTODO}

Se realizó un estudio clínico, observacional, de corte transversal, de pacientes sometidos 
Tabla 1: Pruebas $\chi^{2}(\mathrm{~N}=53)$.

\begin{tabular}{|c|c|c|c|c|c|}
\hline & \multirow[b]{2}{*}{ Valor } & \multirow[b]{2}{*}{ df } & \multicolumn{3}{|c|}{ Significación } \\
\hline & & & $\begin{array}{c}\text { Asintótica } \\
\text { (bilateral) }\end{array}$ & $\begin{array}{c}\text { Exacta } \\
\text { (bilateral) }\end{array}$ & $\begin{array}{c}\text { Exacta } \\
\text { (unilateral) }\end{array}$ \\
\hline$\chi^{2}$ de Pearson & $4.246^{*}$ & 1 & 0.039 & & \\
\hline Corrección de continuidad** & 3.181 & 1 & 0.075 & & \\
\hline Razón de verosimilitud & 4.305 & 1 & 0.038 & & \\
\hline Prueba exacta de Fisher & & & & 0.054 & 0.037 \\
\hline Asociación lineal por lineal & 4.166 & 1 & 0.041 & & \\
\hline
\end{tabular}

a liposucción en la Clínica Berlín, de enero a diciembre de 2017. Se incluyeron a pacientes de ambos sexos a partir de los 18 años, con estado físico de ASA I y II, sometidos a liposucción de más de dos áreas corporales (anterior, posterior, muslos, brazos), incluyendo el tronco, mediante técnica súper húmeda bajo anestesia general balanceada y con registro de valoración postquirúrgica de dolor. Se excluyeron a pacientes sometidos a procedimientos combinados y aquellos con liposucción de un área corporal, así como pacientes sin registro de valoración postquirúrgica del dolor.

Las variables de estudio fueron dolor registrado en dos grupos según la presencia de éste, como «sin dolor» y «con dolor» (EVA > 3) y medicación de rescate, registrando a los pacientes que la solicitaron (EVA $>7$ ).

Los pacientes fueron intervenidos con liposucción convencional, con máquina de aspiración, bajo anestesia general balanceada con sevoflurano 2-2.5 vol\%, fentanilo 0.0026$0.039 \mu \mathrm{g} / \mathrm{kg} / \mathrm{min}$, vecuronio $0.1 \mathrm{mg} / \mathrm{kg}$ (dosis única en inducción), propofol $1.5-2 \mathrm{mg} / \mathrm{kg}$ (dosis única en inducción), con infiltración subcutánea, técnica súper húmeda con solución salina al $0.9 \%$ adicionada con adrenalina $1 \mathrm{mg}$ y el anestésico local (lidocaína $5 \mathrm{mg} / \mathrm{kg}$ y ropivacaína $2 \mathrm{mg} / \mathrm{kg}$, dividido entre el número de soluciones de infiltración de 1 litro empleadas, sin exceder dosis total de $400 \mathrm{mg}$ para el caso de la lidocaína y 200 mg para el
Tabla 2: Tabla cruzada anestésico-dolor.

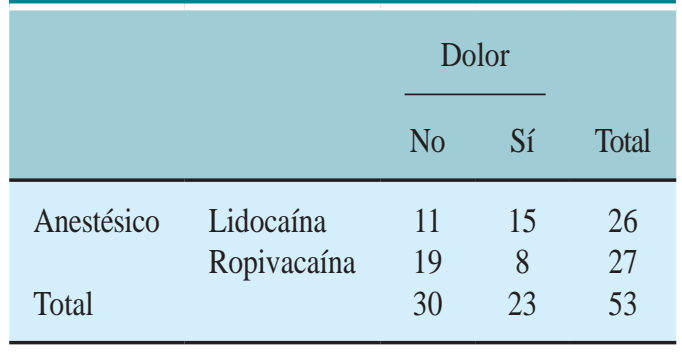

caso de la ropivacaína), con un máximo de 3,000 $\mathrm{mL}$ por paciente.

El esquema analgésico postoperatorio fue con ketorolaco de 30 mg vía intravenosa cada ocho horas, con dosis de rescate de paracetamol de $1 \mathrm{~g}$ vía intravenosa.

Se recolectaron datos en tabla de Excel, agrupados por sexo, edad, estado físico ASA, índice de masa corporal, tipo de anestésico local empleado en soluciones de infiltración, presencia de dolor (dolor $=\mathrm{EVA}>3$ ) durante las primeras 4, 8, 12 y 24 horas postoperatorias. Se registraron los pacientes que requirieron rescate analgésico.

El análisis estadístico se realizó con el programa SPSS versión 20, que incluyó comparación entre los grupos (ropivacaína y lidocaína), con frecuencias simples y relativas en porcentaje para el sexo, edad e índice de masa corporal. Se calculó la incidencia de dolor para cada caso: ropivacaína y lidocaína, la $\chi^{2}$, 
valor de p. De manera adicional, se realizó la prueba log rank sobre el tiempo que transcurrió hasta presentar el evento (EVA >3) con los dos anestésicos locales empleados. Se calculó un modelo de supervivencia de Kaplan-Meier para evaluar el tiempo transcurrido hasta solicitar medicación de rescate (EVA $>7$ ) a las 4 , 8,12 y 24 horas después del procedimiento quirúrgico.

\section{RESULTADOS}

Se incluyeron 53 pacientes con los criterios de selección descritos. Se presentan las características demográficas de los pacientes, sin mostrar diferencias estadísticamente significativas entre ambos grupos con respecto a la edad, sexo e índice de masa corporal (IMC). El promedio de edad del grupo A (lidocaína) fue de $33.8 \pm 8.5$ con un rango de 21 a 54 años y para el grupo B (ropivacaína) de $38.0 \pm 8.6$ con un rango de 23 a 56 años. La media del IMC del grupo A fue de $25.37 \pm 3.92$, con un rango de 19.10 a 33.80 y para el grupo B de $25.80 \pm 4.66$, con un rango de 19.70 a 36.40. La distribución por sexo para el grupo A fue de $84.6 \%$ mujeres y $15.4 \%$ hombres, mientras que para el grupo $B$ fue de $96.3 \%$ mujeres y $3.7 \%$ hombres.

La hipótesis nula establecía que no existe relación entre el dolor y el anestésico local empleado. Para analizar esta hipótesis se empleó un intervalo de confianza del 95\%, con un nivel de significancia del $5 \%$ en la prueba de $\chi^{2}$ con las variables dolor y anestésico local. Como resultado, se obtuvo que la significancia asintótica (bilateral) fue de $0.039<0.05$, y se rechazó la hipótesis nula, es decir, sí existe una relación entre dolor y el anestésico local empleado (Tabla 1), por lo tanto, es estadísticamente significativo. Dado que no existen recuentos con casillas menores a cinco o diferencia significativa entre los totales de filas y columnas, no se tomó en cuenta la prueba exacta de Fisher (Tabla 2).

Se hizo la prueba log rank, con resultado de $p=0.024$, entre los grupos existe una diferencia ante el evento estudiado. Esta diferencia radica en que para el grupo A presenta dolor a partir de las cuatro horas de postoperatorio y para el grupo B los eventos se acotan para las 12 horas en adelante (Figura 1).
Se utilizó la prueba de supervivencia Kaplan-Meier para evaluar la cantidad de horas postquirúrgicas que transcurrieron hasta solicitar medicación de rescate, se obtuvo que para el grupo A la estimación en caso de presentarse el evento fue a las 9.87 horas y para el grupo $\mathrm{B}$ a las 13.50 horas, con una estimación global de 11.13 horas. Mientras que en el grupo A se tiene un error de 1.084 horas, para el grupo $\mathrm{B}$ no se logra estimar debido a que el número de casos que presentaron dolor fue menor al 50\% del total de la muestra (Tabla 3).

\section{DISCUSIÓN}

La importancia clínica en el manejo del dolor postoperatorio en la liposucción se manifiesta en la necesidad de administrar analgésicos de rescate durante el periodo de hospitalización. El dolor se presenta secundario al trauma quirúrgico en las heridas de acceso al tejido celular subcutáneo, así como por el trauma producido por el trayecto de las cánulas de aspiración.

En diversos estudios se ha probado la utilidad de los anestésicos locales para el manejo del dolor postoperatorio. En este estudio se demuestra que los pacientes tratados con ropivacaína tuvieron mejor control analgésico y un tiempo libre de dolor superior a los pacientes tratados con lidocaína, que se traduce en menos dosis de analgésico de rescate y mayor bienestar del paciente. La administración de

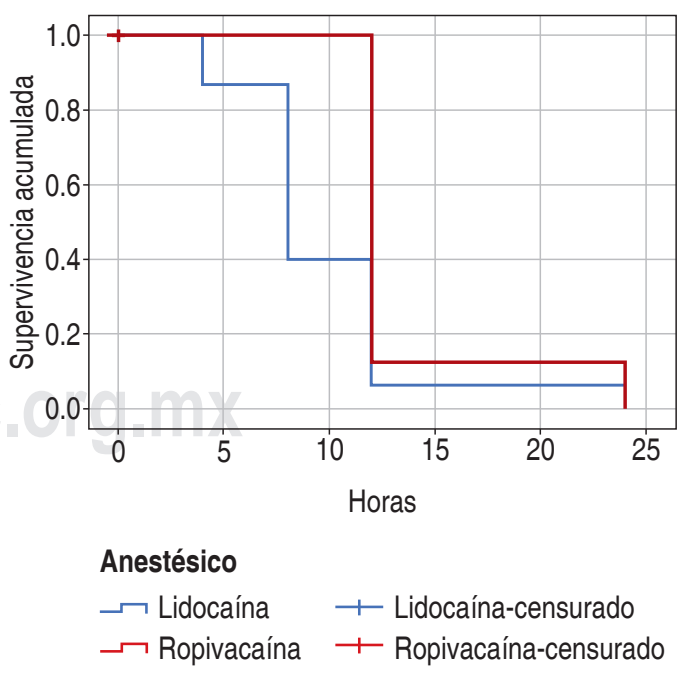

Figura 1: Funciones de supervivencia. 
Tabla 3: Medias y medianas para el tiempo de supervivencia.*

\begin{tabular}{|c|c|c|c|c|c|c|c|c|}
\hline \multirow[b]{3}{*}{ Anestésico } & \multicolumn{4}{|c|}{ Media } & \multicolumn{4}{|c|}{ Mediana } \\
\hline & \multirow[b]{2}{*}{ Estimación } & \multirow[b]{2}{*}{$\begin{array}{l}\text { Desv. } \\
\text { error }\end{array}$} & \multicolumn{2}{|c|}{ IC 95\% } & \multirow[b]{2}{*}{ Estimación } & \multirow[b]{2}{*}{$\begin{array}{l}\text { Desv. } \\
\text { error }\end{array}$} & \multicolumn{2}{|c|}{ IC $95 \%$} \\
\hline & & & $\begin{array}{l}\text { Límite } \\
\text { inferior }\end{array}$ & $\begin{array}{l}\text { Límite } \\
\text { superior }\end{array}$ & & & $\begin{array}{l}\text { Límite } \\
\text { inferior }\end{array}$ & $\begin{array}{l}\text { Límite } \\
\text { superior }\end{array}$ \\
\hline Lidocaína & 9.867 & 1.226 & 7.463 & 12.270 & 8.000 & 1.084 & 5.875 & 10.125 \\
\hline Ropivacaína & 13.500 & 1.500 & 10.560 & 16.440 & 12.000 & - & - & - \\
\hline Global & 11.130 & 1.005 & 9.162 & 13.099 & 12.000 & 0.450 & 11.117 & 12.883 \\
\hline
\end{tabular}

más fármacos implica riesgos y costos extra al manejo programado.

\section{CONCLUSIÓN}

La adición de ropivacaína a las soluciones de infiltración para la cirugía de contorno corporal es una estrategia prometedora para el manejo del dolor postoperatorio. Sin embargo, se requieren más estudios para corroborarlo.

\section{REFERENCIAS}

1. ISAPS Global Statistics 2016. Available in: https://www. isaps.org/medical-professionals/isaps-global-statistics/

2. Klein JA. Tumescent technique for local anesthesia improves safety in large volume liposuction. Plast Reconstr Surg 1993; 92: 1085-1098.

3. Chia C, Neinstein R, Theodorou S. Evidencebased medicine: liposuction. Plast Reconstr Surg 2017; 139 (1): 267e-274e. doi: 10.1097/ PRS.0000000000002859.

4. Matarasso A. Super wet anesthesia redefines largevolume liposuction. Aesthet Surg J 1997; 17: 358-364.

5. Rohrich RJ, Beran SJ, Fodor PB. The role of subcutaneous infiltration in suction-assisted lipoplasty: a review. Plast Reconstr Surg 1997; 99: 514-519.

6. Wells J, Hurvitz K. An evidence-based approach to liposuction. Plast Reconstr Surg 2011; 127 (2): 949-954.
7. Ochoa-Anaya G, Aguirre-Ibarra CP, Franco-Cabrera M. Lidocaína: aspectos generales y nuevas implicaciones en la inflamación. Rev Mex Anest 2017; 40 (3): 220-225.

8. Vincent A, Bernard L, Léone M. Farmacología de los anestésicos locales. EMC - Anestesia-Reanimación 2019; 45 (1): 1-19.

9. Lirk P, Berde CB. Local anesthetic. In: Gropper MA, editor. Miller's anesthesia. 9th ed. Philadelphia: Elsevier; 2020. pp. 865-891.

10. Menezes MVA, Archanjo PT, Oliveira RSR, Dias LAG, Prado JS, Araujo FV et al. Postoperative control of liposuction pain. Rev Bras Cir Plast 2017; 32 (4): 556-561.

11. Kaufman E, Epstein JB, Gorsky M, Jackson DL, Kadari A. Preemptive analgesia and local anesthesia as a supplement to general anesthesia: a review. Anesth Prog 2005; 52 (1): 29-38.

12. Manassa EH, Hellmich S, Ronert $M$, Hofheinz $H$, Olbrisch RR. Pain management after lipoplasty: a study of 303 cases. Plast Reconstr Surg 2005; 115 (6): 1715-1721; discussion 1722.

13. Negus SS. Addressing the opioid crisis: the importance of choosing translational endpoints in analgesic drug discovery. Trends Pharmacol Sci 2018; 39 (4): 327-330.

Conflicto de intereses: Los autores declaran no tener conflicto de intereses.

Correspondencia:

Dra. Sonia Leslie Fuentes-Trejo

E-mail: lesliefuntrav@gmail.com 